\title{
May Updates: People and Places
}

\begin{abstract}
AALAC awards US Fellowship
AAALAC International has named

Michelle Wallace-Fields as the 2018 US

recipient of its annual Fellowship Award.

Wallace-Fields is the Associate Director

of Husbandry for the Office of Laboratory

Animal Resources at the University of

Colorado Denver in Aurora, Colorado; she

is also the liaison to the Environmental

Health and Safety Group, Institutional

Biosafety Committee, and the IACUC.

The US Fellowship is awarded to an

AALAS-registered individual in recognition

of their contributions to laboratory animal

care and use.
\end{abstract}

\section{Commendable papers}

The NC3Rs held their annual International Prize Ceremony on March $12^{\text {th }}$ in London. The prize, sponsored by GlaxoSmithKline, consists of a $£ 28 \mathrm{k}$ grant and $£ 2 \mathrm{k}$ personal award for the publication of a scientific or technological advance in the 3Rs. The 2017 Prize went to Elisa Passini from the University of Oxford, for her 2017 paper in Frontiers in Physiology describing the development of an in silico model of cardiac drug toxicity that was more accurate than animal models.

Two highly commended papers each received a $£ 4 \mathrm{k}$ grant and $£ 1 \mathrm{k}$ personal award. Christian Tiede from the University of Leeds was commended for his 2017 paper published in eLife on affimer proteins, a potential alternative to animal-made antibodies. Michael Walker from the University of Guelph was also commended for his 2016 BMC Medical Research Methodology paper describing split-plot experimental designs that can reduce the number of animals needed for a study by more than half while still achieving $80 \%$ power.

\section{Surgery expands out west}

New Jersey-based preclinical contract research organization Envigo has announced plans to expand its research model surgical facility in Livermore, California. The facility, which produces translational surgical models, will grow from 500 square feet to 5,000, employ double the current number of surgical technicians, and include advanced equipment such as intensive care unit recovery chambers, ventilator and microscopic technologies, and RFID and surgical tracking systems. Head of North American Surgery Brad Gien explained in a press release that the expansion is in response to increased demand from biopharmaceutical customers on the west coast and will complement the company's existing surgical facility in Indianapolis, Indiana. "Accordingly, we are now able to service a greater volume of orders given the more efficient logistics to

\section{Careers update}

On August 1, 2018, Penn Veterinary Medicine at the University of Pennsylvania will welcome a new dean. Andrew Hoffman will succeed Joan Hendricks, dean since 2006, as the next Gilbert S. Kahn Dean at the veterinary school in Philadelphia. Hoffman joins Penn Veterinary Medicine from Tufts University Cummings School of Veterinary Medicine; he joined Tufts in 1994 as an assistant professor and served as director of various divisions over the years, including the Lung Function Testing Laboratory, the Stem Cell Laboratory, and since 2012, the Regenerative Medicine Laboratory.

He received his Doctorate of Veterinary Medicine degree from Cornell University in 1985 and a Doctor of Veterinary Science degree in pulmonology from the University of Guelph in 1990. His research interests have included respiratory physiology, the development of stem cell treatments, and regenerative medicine, with a One Health approach.

Penn President Amy Gutmann commented in a press release, Hoffman "has a long and distinguished track record and an exciting vision for the role of veterinarians and veterinary schools in research universities and society."

service our customers in the region," he says. The facility is expected to open later this spring.

\section{Taconic's SAB}

Rensselaer, New York-based mouse model provider Taconic has announced the formation of a Scientific Advisory Board (SAB). The $\mathrm{SAB}$ will provide scientific guidance to management. CEO Bob Rosenthal commented in a press release, "Discovery and pre-clinical animal models continue to move toward highly-complex and high-value translational models.

To continue to provide the best solutions for our customers, it is essential for Taconic to have guidance from prominent scientific experts as we develop and validate new models and services."

The trio selected includes J. Victor Garcia from the University of North Carolina at Chapel Hill, with experience in infectious disease research; microbiologist Andrew Goodman from Yale University School of Medicine; and pharmacologist David Hill, who has prior work experience with pharmaceutical companies and scientific expertise in the areas of neuroscience, pain, anesthesia, cardiometabolic disease, and oncology.

\section{Brain Prize}

Four Alzheimer's disease researchers have been awarded this year's one million euro Brain Prize from the Lundbeck Foundation in Denmark. The Prize has been awarded annually since 2011 to active researchers in recognition of their contributions to the field of neuroscience. The 2018 winners include: John Hardy, University College London Institute of Neurology; Christian Haass, German Center for Neurodegenerative Diseases, DZNE in Munich; Bart De Strooper, UK Dementia Research Institute at University College London; and Michel Goedert, Laboratory for Molecular Biology of the Medical Research Council in Cambridge, UK. They will receive the award at a ceremony in Denmark on May 9. 